\title{
Neuropsychological function and brain arteriovenous malformations: redefining eloquence as a risk for treatment
}

\author{
Ronald M. Lazar, Ph.D. \\ Departments of Neurology and Neurological Surgery, Columbia University College of Physicians and \\ Surgeons, New York, New York
}

\begin{abstract}
Arteriovenous malformations (AVMs) of the brain, thought to begin during fetal development and undergo an unclear maturation process; most often do not become symptomatic until the patient reaches adulthood. Authors of recent prospective studies have suggested that relatively few patients present with neuropsychological abnormalities in the absence of hemorrhage. In general, neuropsychological evaluations in patients with AVMs have yielded mixed results with respect to localization of function, and the authors of most cognitive studies have not been able to demonstrate the nature and degree of impairment seen in acute ischemic stroke in comparable brain regions. Superselective Wada testing prior to therapeutic embolization, functional imaging studies, and intrasurgical cortical mapping have shown redistribution of language and memory to unpredictable regions. Developmental cognitive history in these patients indicates that most will have at least some background of learning problems during the school-age years with varying degrees of severity, reflecting a time when brain reorganization may be occurring. These data suggest that traditional assumptions about the eloquence of brain regions may not hold for patients with AVMs and that establishing treatment risk in the individual patient needs to be made on an empirical basis.
\end{abstract}

\section{KEY WORDS • arteriovenous malformation - functional imaging • neuropsychological function • superselective Wada testing}

Arteriovenous malformations of the brain are lesions composed of a coiled batch of arteries and veins, joined by arteriovenous fistulas and partially separated by sclerotic tissue. Although lying in a bed formed by displacement rather than invasion of normal brain tissue, AVMs are pulsatile vascular masses that impact on brain hemodynamics and physiology in ways not yet fully identified. ${ }^{1}$ Arteriovenous malformations appear to begin forming in utero and undergo an unclear "maturation" process for many years until some critical mass or event is reached and symptoms, usually seizures or hemorrhage, ensue. ${ }^{27}$ More than $80 \%$ of cerebral AVMs become symptomatic after the patient reaches the age of 15 years. ${ }^{21}$ Thus, AVMs represent a unique class of lesions whose full range of clinical manifestations, including neuropsychological function, are just beginning to be understood.

For the purposes of this review, "neuropsychological function" refers to the complex set of behavioral tasks subserved by the brain involving such spheres as language, memory, attention, visual perception and executive skills. ${ }^{16}$ The terms "neuropsychological" and "cognitive" are used here interchangeably. It has been suggested that

Abbreviations used in this paper: $\mathrm{AVM}=$ arteriovenous malformation; fMR = functional magnetic resonance. the assessment of cognitive function is probably a more sensitive index of brain integrity in cerebrovascular disease than sensorimotor function or coordination. ${ }^{10,22}$ The relevance of neuropsychological function to the study of AVMs in particular lies in three spheres: understanding the natural history, assessing clinical manifestations of hemorrhage from AVMs, and calculating treatment risk.

The short- and long-term effects on surrounding tissue of blood flow through an AVM are not well understood and are thus still a matter of debate. One theoretical but as yet unproved paradigm that some still consider an explanation for deficits in untreated AVMs is the notion of steal. ${ }^{5}$ The results of recent prospective studies, however, have largely disproved the notion that shunt-induced reduction in perfusion in the vascular territories surrounding an AVM produces functional deficits. In one study, for example, 152 consecutive patients with cerebral AVM were studied for evidence of focal neurological deficits unrelated to hemorrhagic events. ${ }^{20}$ It was found that only two patients demonstrated a progressive neurological syndrome and only seven had static focal signs, of whom only three had a disorder of neuropsychological function. There are, however, no large prospective series of AVMs in which evaluation of cognitive function over time has been studied. 


\section{NEUROPSYCHOLOGICAL ASSESSMENT}

Neuropsychological investigations of patients with AVM have yielded mixed results with respect to localization of function, and most neurocognitive studies have not been able to demonstrate the kinds of impairment typically seen with acute focal lesions in comparable brain regions. Waltimo and Putkonen ${ }^{30}$ conducted one of the earliest studies of the cognitive sequelae of AVMs by using well-validated measures. They found that the average intelligence quotient of 40 patients with AVM did not differ from the normal population. Moreover, no differences were revealed on any of the neuropsychological instruments between individuals with left- or right-sided lesions. Even the association between previous hemorrhage and cognitive impairment did not reach statistical significance.

Brown and colleagues ${ }^{4}$ assessed the extent to which neuropsychological tests could predict the laterality of angiographically confirmed AVMs compared with the laterality of ischemic stroke. The discriminant function analysis showed that neuropsychological measures were related to the hemispheric side on which an ischemic stroke had occurred, but not the laterality of an AVM. Similarly, Mahalick and coworkers ${ }^{17}$ studied 24 patients with AVMs prior to surgical treatment. Compared with 24 matched normal control volunteers, patients with AVMs showed variable degrees of impairment in verbal and visuospatial function. More important, the cognitive deficits detected were associated with the hemisphere contralateral to the side with the AVM. Steal was posited as the causal mechanism for these findings but with no physiological data to support this hypothesis. It has been proposed by others, however, that that one possible reason for the variability of neuropsychological impairment seen among patients with AVMs could be atypical organization of function. ${ }^{3}$

The failure to find evidence supporting steal as a frequent cause of AVM-related deficits and the suggestion of unusual cerebral localization of neurocognitive function in neuropsychological testing raise questions that pertain to AVM treatment risk. One factor in the grading of such risk has been the so-called eloquence of adjacent brain tissue. ${ }^{26}$ In the original design calculation of the SpetzlerMartin grade, eloquent areas were defined as sensorimotor, language, visual cortex, hypothalamus, internal capsule, brainstem, cerebellar peduncles, and deep cerebellar nuclei. Spetzler and Martin assumed that "eloquent cortical regions occupy their normal anatomic location." Recent data derived from the use of highly sophisticated combinations of radiological, surgical, and behavioral techniques now indicate that this assumption may longer be correct.

\section{SUPERSELECTIVE WADA TESTING FOR ELOQUENT FUNCTION}

Prior to conventional surgery or radiosurgery, many patients are treated by angiographically guided injection of embolic material into arteries supplying the AVM that will reduce flow, ultimately producing thrombosis. ${ }^{31}$ Before superselective injection of glue, a frequently used thrombogenic agent at many centers, it is common practice to first determine whether the tip of the catheter has been placed inadvertently proximal to the nidus of the AVM so that permanent closure might affect eloquent function, such as language, memory, vision, and motor control. ${ }^{6,15,24,25}$ This preembolization assessment is usually composed of injection of an anesthetic agent along with a contrast agent in a procedure that is based on the technique of Wada and Rasmussen of injecting amobarbital sodium into the internal carotid arteries of patients with epilepsy to determine cerebral dominance for language and memory prior to surgery. ${ }^{29}$ During such testing for eloquent brain regions in patients with AVM prior to embolization, the injection of anesthetic agents is performed in arterial pedicles distal to the circle of Willis.

One of the earliest reports was a case study in which a patient with a left posterior temporal AVM underwent speech-evoked magnetoencephalography, superselective Wada testing, and direct electrical stimulation of the cortex during surgery. ${ }^{19}$ Except for some reading errors, the clinical examination of language was normal. The topographic map of the magnetic component to stimulation with auditory syllables showed a source that was displaced superiorly to the probable location in the auditory cortex. Superselective Wada testing showed no deficit in receptive or expressive language with selective injection of the posterior temporal branch into superior temporal gyrus, and naming and repetition were not affected during stimulation at seven of eight usual language sites in the temporal and inferior parietal cortex in the vicinity of the AVM. During the postoperative course the patient displayed a mild expressive aphasia, which fully resolved 2 weeks later.

Lazar, et al., ${ }^{9}$ took advantage of the superselective Wada procedure in AVM patients to address possible redistribution of language. It was found that when right-handed adult patients with left temporoparietal AVM underwent selective anesthetic injections into the left prefrontal region, all of them developed major language symptoms including loss of comprehension, fluent speech with paraphasias, and perseveration that resembled Wernicke aphasia. To address the cerebral localization of language in patients with left frontal AVMs, three patients underwent the same language evaluation as those who harbored the posterior AVMs. ${ }^{11}$ Anesthetic injection into the vessels supplying the inferior division of the left middle cerebral artery produced the expected Wernicke area, verifying left cerebral dominance for language in each case. Superselective study in the left frontal regions, however, resulted in mild right-sided paresis in all patients, but no language abnormality and there was no loss of fluency. Embolization of vessels feeding the AVM then took place without significant morbidity in a region where traditional assumptions of eloquence might have precluded treatment.

Preembolization superselective Wada testing has also shown unpredictable redistribution of other cognitive functions. ${ }^{12}$ In this small case series, in one patient with a left parietal AVM and a right temporal AVM oral reading was controlled by the right hemisphere and reading comprehension was subserved by the left hemisphere. A patient with a left medial AVM displayed the presence of an anterograde amnesia and alexia but not aphasia with a left superior temporal artery injection. 


\section{FUNCTIONAL IMAGING}

Similar findings of unpredicted cerebral localization have also been seen with functional imaging. Leblanc, et al. ${ }^{14}$ reported the first case of a patient with an angiographically proven AVM in the left midposterior second convolution who underwent positron emission tomography scanning while performing language tasks. In addition to activation in the Wernicke and Broca regions, there was also increased metabolism in the right temporal region. This same group described 10 patients with leftsided AVM who underwent positron emission tomography investigation, one of whom had an AVM in the left superior temporal area and whose functional language activation was located entirely in the right hemisphere. ${ }^{13}$

Functional magnetic resonance imaging has also been performed in patients with AVM. Latchaw, et al., ${ }^{7}$ reported on two of three patients in whom the AVM was located in traditionally language-sensitive regions. In the case of a left frontotemporal AVM, an fMR imaging language activation using a silent word generation paradigm, revealed that speech cortex was located anterior and deep to the AVM. A patient with a left temporal AVM also demonstrated silent speech activation anterior to the AVM. More recently Maldjian, et. al. ${ }^{18}$ studied two patients with temporal lobe AVMs and found bilateral temporal lobe activation, but similar activation was also found in the resting condition. Two patients with left anteroinferior frontal AVMs demonstrated activation in the left inferior frontal lobe as well as in the superior left frontal lobe anterior to the AVM nidus. More recently, Vinkingstad, et al., ${ }^{28}$ evaluated language tasks during fMR imaging prior to surgery in four patients with left cerebral AVMs, revealing substantial right hemisphere activation in each case.

The problem with the use of functional imaging to determine, for example, critical language regions in the brain is that are few data from such studies showing that "activated" brain structures are necessary for language to take place. ${ }^{2}$ Indeed, an attempt was made to correlate findings on fMR imaging language activation with superselective Wada testing. ${ }^{11}$ A silent speech task in a right-handed patient with a Broca area AVM produced fMR imaging activation in the contralateral right frontal opercular region and posterior to the AVM adjacent to the rolandic fissure. With the fMR imaging data alone, it might have been concluded that the patient had developed a new language region in the left hemisphere, but the anesthetic injection into the arterial supply for the fMR imaging- activated rolandic region failed to produce any language abnormality. More research with functional imaging is therefore needed to establish activated regions that are important for a task or merely represent epiphenomena. In the meantime, Wada testing remains the only model capable of establishing sites biologically necessary for a function prior to craniotomy.

\section{COGNITIVE HISTORY AND AVM}

Most of the knowledge regarding cortical localization of neuropsychological function comes from cerebral infarction; the most common cause of aphasia, for example, is stroke. ${ }^{23}$ In contrast to such acute events, however, brain AVMs are thought to be the result of vascular dysgenesis during fetal development and appear to develop over many years. The question arises, then, as to when brain reorganization occurs in these patients who are usually otherwise asymptomatic until their 30 s and 40s.

As a first step toward addressing this matter, Lazar and colleagues ${ }^{8}$ used structured telephone interviews to compare developmental history in adult patients with AVM with a group of other patients with other chronic intracranial lesions. There were 44 randomly selected patients with AVM and 32 comparison patients: 15 randomly chosen patients from the institution's database of cerebral aneurysms and all 17 patients who underwent a biopsy over a 5-year period with a diagnosis of low-grade tumor. Queries were made as to education, special classes, classification of learning disabilities, failing grades, as well as if there had been any experience of significant problems in skill areas such as reading, writing, and attention. Of the 44 AVM patients, 11 presented with hemorrhage but none presented before 20 years of age. There was no difference in the age, education, or report of delayed developmental milestones (speech or motor function) between the AVM patients and other patients. In contrast, 33 patients with AVM (75\%) reported a positive occurrence to any survey question, with $66 \%$ of AVM patients indicating that they had experienced difficulty with at least one skill area during their school years. The tumor and aneurysm patients were no different from the general population in reporting these problems. The importance of these findings is that despite the likely presence of some form of a primitive AVM at birth, there may not have been any functional consequences before the age of 3 or 4 years. Although retrospective in nature, these are among the first data to shed some insight on the time course of AVM development.

\section{CONCLUSIONS}

In summary, the assessment of neuropsychological function in AVM patients in the office, in the angiography suite, and on the operating room table has shown a degree of cerebral redistribution not found or studied as thoroughly to date in any other neurological disease. Further research is needed to determine whether functional reorganization in AVM patients can serve as a model for adaptation in other chronic brain conditions. Nevertheless, the data show that traditional assumptions about the importance of brain regions in these patients do not hold, at least for cognitive function, so that establishing treatment risk in any one patient based on so-called eloquence needs to be made empirically.

\section{References}

1. Anonymous: Arteriovenous malformations in the brain in adults. N Engl J Med 340:1812-1818, 1999

2. Binder JR, Frost JA, Hammeke TA, et al: Human brain language areas identified by functional magnetic resonance imaging. J Neurosci 17:353-362, 1997

3. Brown GG, Spicer KB, Malik G: Neurobehavioral correlates of arteriovenous malformations and cerebral aneurysms, in Bornstein RA, Brown GG (eds): Neurobehavioral Aspects of Cerebrovascular Disease. New York: Oxford University Press, 1991, pp 202-223

4. Brown GG, Spicer KB, Robertson WM, et al: Neuropsycholog- 
ical signs of lateralized arteriovenous malformations: comparison with ischemic stroke. Clin Neuropsychol 3:340-352, 1989

5. Hachinski V, Norris JW, Cooper PW, et al: Symptomatic intracranial steal. Arch Neurol 34:149-153, 1977

6. Han MH, Chang KH, Han DH, et al: Preembolization functional evaluation in supratentorial cerebral arteriovenous malformations with superselective intraarterial injection of thiopental sodium solution. Acta Radiol 35:212-216, 1994

7. Latchaw RE, Hu X, Ugurbil K, et al: Functional magnetic resonance imaging as a management tool for cerebral arteriovenous malformations. Neurosurgery 37:619-626, 1995

8. Lazar RM, Connaire K, Marshall RS, et al: Developmental deficits in adult patients with arteriovenous malformations. Arch Neurol 56:103-106, 1999

9. Lazar RM, Marshall RS, Pile-Spellman J, et al: Anterior translocation of language in patients with left cerebral arteriovenous malformation. Neurology 49:802-808, 1997

10. Lazar RM, Marshall RS, Pile-Spellman J, et al: Continuous time estimation as a behavioral index of human cerebral ischaemia during temporary occlusion of the internal carotid artery. J Neurol Neurosurg Psychiatry 60:559-563, 1996

11. Lazar RM, Marshall RS, Pile-Spellman J, et al: Interhemispheric transfer of language in patients with left frontal cerebral arteriovenous malformation. Neuropsychologia 38:1325-1332, 2000

12. Lazar RM, Marshall RS, Pile-Spellman J, et al: Unpredicted redistribution of higher cerebral function in patients with AVM. Neurology 48:A292, 1997 (Abstract)

13. Leblanc E, Meyer E, Zatorre R, et al: Functional PET scanning in the preoperative assessment of cerebral arteriovenous malformations. Stereotact Funct Neurosurg 65:60-64, 1995

14. Leblanc R, Meyer E, Bub D, et al: Language localization with activation positron emission tomography scanning. Neurosurgery 31:369-373, 1992

15. Lee GP, Meador KJ, Murro, AM, et al: Amobarbital evaluation of neurobehavioral function prior to therapeutic occlusion of brain arteriovenous malformations: a new neuropsychological procedure, Appl Neuropsychol 3:1-7, 1996

16. Lezak MD: Neuropsychological Assessment, ed 3. New York: Oxford University Press, 1995

17. Mahalick DM, Ruff RM, U HS: Neuropsychological sequelae of arteriovenous malformations. Neurosurgery 29:351-357, 1991

18. Maldjian J, Atlas SW, Howard RS II, et al: Functional magnetic resonance imaging of regional brain activity in patients with intracerebral arteriovenous malformations before surgical or endovascular therapy. J Neurosurg 84:477-483, 1996

19. Martin NA, Beatty J, Johnson RA, et al: Magnetoencephalography localization of a language processing cortical area adjacent to a cerebral arteriovenous malformation. Case report. J Neurosurg 79:584-588, 1993

20. Mast H, Mohr JP, Osipov A, et al: 'Steal' is an unestablished mechanism for the clinical presentation of cerebral arteriovenous malformations. Stroke 26:1215-1220, 1995

21. Millar C, Bissonnette B, Humphreys RP: Cerebral arteriovenous malformations in children. Can J Anaesth 41:321-331, 1994

22. Mohr JP: Thomas Willis Lecture. Acute clinical trials: an expression of concern. Cerebrovasc Dis Suppl 3:45-50, 1999

23. Post-Stroke Rehabilitation Guideline Panel: Post-Stroke Rehabilitation/Post-Stroke Rehabilitation Guideline Panel. Rockville, MD: US Department of Health and Human Services, 1995

24. Rauch RA, Viñuela F, Dion J, et al: Preembolization functional evaluation in brain arteriovenous malformations: the ability of superselective Amytal test to predict neurologic dysfunction before embolization. AJNR 13:309-314, 1992

25. Rauch RA, Viñuela F, Dion J, et al: Preembolization functional evaluation in brain arteriovenous malformations: the superselective Amytal test. AJNR 13:303-308, 1992

26. Spetzler RF, Martin NA: A proposed grading system for arteriovenous malformations. J Neurosurg 65:476-483, 1986

27. Stein BM, Pile-Spellman J, Isaacson SR: Vascular malformations of the brain and dura, in Barnett HJM, Mohr JP, Stein BM, et al (eds): Stroke: Pathophysiology, Diagnosis, and Management. New York: Churchill-Livingston, 1998, pp 1309-1338

28. Vikingstad EM, Cao Y, Thomas AJ, et al: Language hemispheric dominance in patients with congenital lesions of eloquent brain. Neurosurgery 47:562-570, 2000

29. Wada J, Rasmussen T: Intracarotid injection of sodium amytal for the lateralization of cerebral speech dominance: experimental and clinical observations. J Neurosurg 17:266-282, 1960

30. Waltimo O, Putkonen AR: Intellectual performance of patients with intracranial arteriovenous malformations. Brain 97: 511-520, 1974

31. Young WL, Pile-Spellman J: Anesthetic considerations for interventional neuroradiology. Anesthesiology 80:427-456, 1994

Manuscript received July 30, 2001.

Accepted in final form August 9, 2001

The preparation of this manuscript was supported in part by PHS Grant NS40792-01 from the National Institute of Neurological Disorders and Stroke.

Address reprint requests to: Ronald M. Lazar, Ph.D., Neurological Institute, 710 West 168th Street, New York, New York 10032. email:ral22@columbia.edu. 